\title{
Aspectos químicos y estructurales de la matriz cementante utilizada en la cultura maya
}

\author{
H. Díaz-Coutiño ${ }^{1}, H$. Ramírez de Alba ${ }^{1}$ y R. Pérez-Campos ${ }^{2}$ \\ ${ }^{1}$ Facultad de Ingeniería CU-UAEM Toluca, Edo. de México e \\ 2 Instituto Mexicano del Petróleo \\ E-mail: rcamposp@imp.mx
}

(recibido: diciembre de 2000, aceptado: abril de 2001)

\begin{abstract}
Resumen
En esta investigación se presentan algunos resultados sobre la composición química y estructural de la matriz cementante ampliamente utilizada en las construcciones del mundo Maya. Para este objetivo se analizaron diferentes tipos de materiales tales como estucos, morteros y concretos que provienen de diferentes lugares, principalmente de Palenque, Yaxchilán y Toniná. Se presentan también comparaciones con los cementos Portland utilizados actualmente. Las composiciones químicas se obtuvieron mediante técnicas de microscopía electrónica de barrido y las caracterizaciones estructurales utilizando técnicas de difracción de rayos X. La determinación de óxidos en estos materiales utilizaron técnicas de espectroscopía de arco y chispa.
\end{abstract}

Descriptores: composición química, composición estructural, matriz cementante, materiales.

\begin{abstract}
In this investigation some results on the chemical composition and also structural characterization of the cement matrix widely used in the constructions of the Mayan world are presented. Different materials such as: stuccos, mortars and concretes which were collected from different Mayan cities such as: Palenque, Yaxchilán and Toniná were analyzed. These results were compared with those obtained from commercial Portland cements. The chemical compositions were obtained using scanning electron microscopy techniques, however the structural characterizations were based on X-ray diffraction studies. The oxide compositions of these materials were obtained using an arc and spark spectroscopy.
\end{abstract}

Keywords: chemical composition, structural composition, gluing matrix, materials.

\section{Introducción}

La búsqueda de materiales durables y resistentes a lo largo de las diversas etapas históricas, ha sido y es, preocupación constante de toda cultura, ya que permiten realizar construcciones para satisfacer requerimientos de vivienda, así como infraestructura para la producción y soporte de la organiza. ción social, religiosa y militar. Existen culturas que solamente han podido ser reveladas por el estudio de sus restos, entre ellos, los de sus construcciones. Dentro de los materiales más usuales y durables destacan la piedra y los materiales termo remanentes (cementantes, arcilla cocida etc.). Se identifican también dos pasos trascendentales en la historia de la construcción; el primero, juntar elementos constructivos, y el segundo, la elaboración de piedras artificiales de formas convenientes para formar estructuras durables y resistentes. Lo anterior ha dado como resultado dos importantes tecnologías: la de los morteros y mamposterías y la del concreto. Generalmente es aceptado que estas tecnologías tuvieron su origen en las culturas griega y romana; sin embargo, otras culturas de manera independiente lograron desarrollos similares, tal es el caso de la civilización Maya que con su tecnología de materiales de construcción, logró admirables conjuntos urbanos y religiosos.

En esta investigación, se presenta un panorama general de los logros de la cultura Maya sobre las técnicas y materiales utilizados en la construcción de sus grandes ciudades. Este estudio está basado principalmente en las propiedades químicas y mecánicas de muestras provenientes de distintas zonas arqueológicas. 
El lugar de origen de esta gran civilización se ubica en las "tierras bajas" del territorio Guatemalteco, la región del Petén, zona selvática y de lluvias torrenciales, cuyo suelo calcáreo y sembrado de núcleos de sílice es apenas cubierto por una delgada capa de tierra cultivable. Ante estas características tan hostiles para la sobrevivencia humana, resulta paradójico el hecho de que precisamente ahí fuera el lugar de nacimiento de una de las culturas más grandes de la América Precolombina. Algunos investigadores sitúan el período formativo preclásico 1500 A.C. hasta 317 D.C., el clásico desde 317 hasta 900 D.C., y un período post-clásico de 900 hasta 1697 D.C. (Morley, 1972; Soustelle, 1990). En este artículo como ya se mencionó, se tratarán algunos de los materiales utilizados en la ingeniería de la construcción, en particular, se mostrarán algunos resultados obtenidos del estudio de materiales tales como estucos, morteros y concretos. Las construcciones Mayas se pueden clasificar en función de su uso, algunos tipos se describen en forma general a continuación:

\section{Desarrollo de las construcciones mayas}

\section{Construcciones administrativas y de carácter religioso}

No se pretende analizar los estilos arquitectónicos de las construcciones (Puuc, Chenes, Río Bec, etc.) porque este campo corresponde a los especialistas, únicamente se mencionan para marcar su diferencia, ya que corresponden a las construcciones más grandes y majestuosas de la ingeniería Maya, las cuales se cuentan por cientos y quizá miles. Las figuras 1 y 2 muestran dos ejemplos típicos de estas construcciones. En este grupo de construcción están comprendidas las grandes pirámides de la ciudad de Tikal Guatemala que alcanzan alturas de $74.0 \mathrm{~m}$, el templo de las inscripciones en Palenque, Chiapas, ciudad Maya famosa por la belleza y calidad de sus tableros de estuco, y el gran palacio del gobernador en Uxmal, Yucatán que en su fachada contiene 20000 piedras talladas y perfectamente ensambladas.

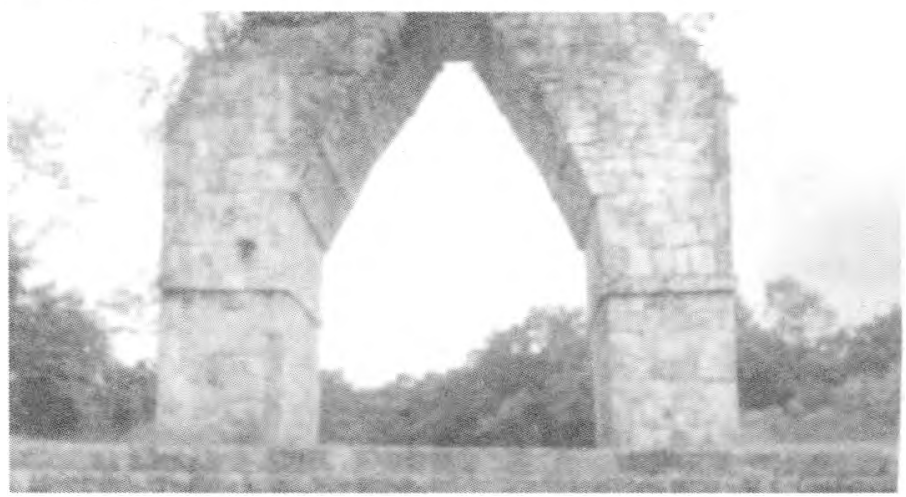

Figura 1. Arco maya construido a base de losas saledizas

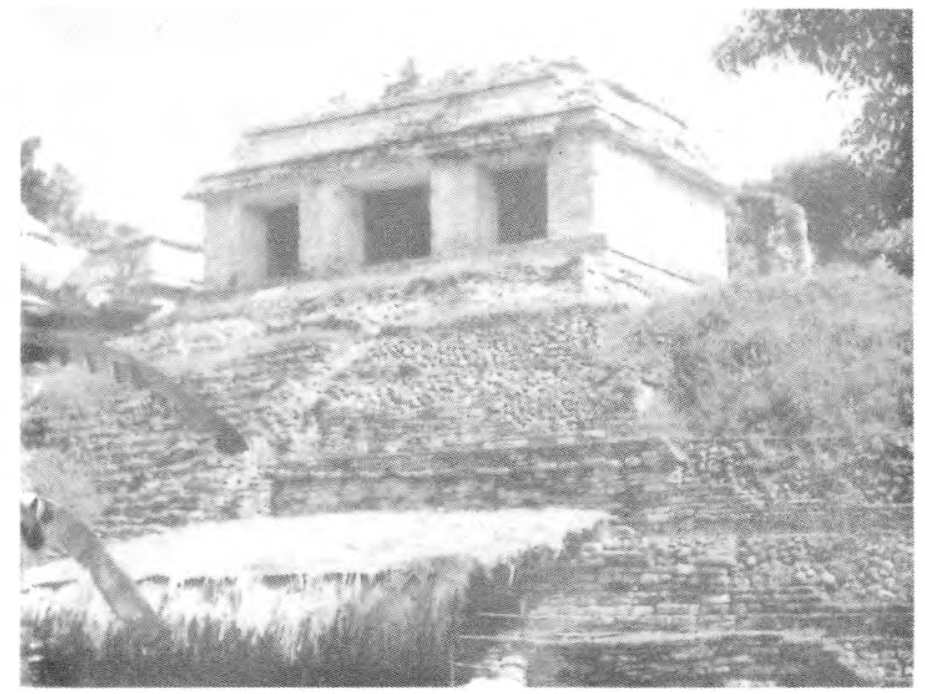

Figura 2. Ejemplo de construcción Maya

\section{Construcciones deportivas}

Estas construcciones son comunes en la mayoría de las culturas prehispánicas, confirmando de paso, la gran relación que existía entre ellas. Eran espacios rectangulares (verdaderos estadios) con marcadores adosados a muros, donde los jugadores anotaban sus "puntos", estos marcadores generalmente eran piedras bellamente talladas. Actualmente, estos espacios bien diseñados y construidos se les conoce como el "juego de pelota" y eran parte muy importante en la planeación y ejecución de las ciudades Mayas.

\section{Viviendas}

No está perfectamente definido si en los palacios Mayas, habitaba la clase gobernante y sacerdotal, lo cual sería lo más lógico. Esta duda cabe, por lo poco funcional de los espacios en dichas construcciones, pero es un hecho que el pueblo común habitaba en viviendas sencillas muy parecidas a las actuales, utilizadas comúnmente en la región del sureste mexicano. Estas viviendas se construyeron, hincando primeramente unos puntales de madera (horcones), después se colocaban las vigas de madera para posteriormente construir las paredes con un entramado de varillas delgadas y flexibles (de madera); finalmente, aplicaban un entortado de barro con zacate picado para darle ductilidad. El techo se construye con un entramado de varillas y lechos de zacate o palma, que por lo general, consta de dos o más aguas.

\section{Construcciones astronómicas}

En Uaxactún se descubrió el observatorio astronómico más antiguo, éste era parte muy importante en la planeación de todas las ciudades, y así debía ser, pues de las observaciones llevadas a cabo se deducían los tiempos mejores para la 
siembra del maíz, alimento básico pero no único en la dieta Maya. Estos observatorios astronómicos de sección transversal cilíndrica y rematados por una cúpula, como puede observarse en Chichen-Itzá, tiene una forma muy parecida a los actuales. Éstos cumplieron muy bien su función, pues sus resultados pueden comprobarse en la exactitud del calendario Maya (Morley, 1972) y el cálculo del tiempo (período) de cada lunación, así como el ciclo de Venus (Xux-eK, la estrella avispa), del cual conocieron perfectamente el período de su revolución sinódica.

\section{Construcciones hidráulicas y sanitarias}

El agua es un elemento vital para cualquier cultura. La mayoría de las ciudades Mayas estaban asentadas en regiones donde no existían ríos, por esa razón el almacenamiento del liquido se realizaba en cisternas (chultunes) distribuidas en las ciudades. Éstas eran depósitos semiesféricos construidos bajó el terreno y estaban revestidos de mortero basado en cal. Tenían una capacidad de 28000 litros, aunque en las grandes ciudades como el Tikal por ejemplo, estos chultunes eran grandes depósitos con suficiente capacidad para abastecer permanentemente de 2000 a 6000 personas (Morley, 1972 y Rivera, 1985). Los chultunes tenían en la parte superior un pequeño orificio y el piso tenía las pendientes y formas adecuadas para captar toda el agua de lluvia que fuera posible, como una especie de embudo. Los ingenieros mayas también construyeron sistemas de drenaje y alcantarillados, por ejemplo, en Comalcalco. También se puede señalar el acueducto en Palenque, construido básicamente en vigas de piedra (Soustelle, 1990). Por otro lado, los Mayas utilizaban la agricultura peraltada y en las márgenes del río Candelaria en Campeche, se observaban aún miles de hectáreas con un perfecto sistema de riego a base de una retícula de canales muy bien trazados, cuyo origen se supone fue basado en los desarrollos Mayas.

\section{Caminos (Sacbé, camino blanco)}

El sistema de caminos Mayas era amplio y efectivo, estos caminos no tuvieron las grandes longitudes de los Incas o Romanos; sin embargo, su anchura y sistema constructivo los hacen ser de los mejores. La anchura varia desde 4.5 hasta 16 metros. Son verdaderos muros rectos levantados a nivel del suelo, con una altura mínima de 0.50 hasta 2.50 metros, atraviesan selvas y pantanos sin interrupción y están perfectamente trazados. Eran construidos alineando dos muros paralelos de mampostería (piedra y mortero) que luego se rellenaban de piedra y cascajo (Morley, 1972 y Rivera, 1985), se remataban con una buena carpeta de mortero (sascab), aunque algunas veces, en lugar de carpetas utilizaban losas ligadas con mortero. El camino más largo que se conoce es el que comunica Cobá y Yaxuná con una extensión de $100 \mathrm{Km}$. con una anchura promedio de $4.5 \mathrm{~m}$. Al respecto, se localizó un rodillo de piedra con una longitud de $4 \mathrm{~m}$, un diámetro de $0.65 \mathrm{~m}$ y un peso de 5 toneladas. Se estima que era un compactador de suelos y que se necesitaron 15 hombres para desplazarlo; en la actualidad no se han encontrado más instrumentos de este tipo.

\section{Puentes}

Como se expresó anteriormente, las grandes ciudades Mayas fueron construidas generalmente en regiones carentes de ríos, con algunas excepciones, entre las que se pueden mencionar Yaxchilán, Chiapas y Copán en la república de Honduras. La primera se sitúa a orillas del río Usumacinta y la segunda a orillas del río Copán. En Yaxchilán en plenas aguas del río Usumacinta, se encuentran vestigios de lo que pudo ser una de las pilas de un gran puente. Se han escrito diversos artículos al respecto, de los cuales el de James O'kon (1995) fue el más interesante por los argumentos que maneja, así como los cuidadosos estudios que llevó a cabo. De acuerdo con O'kon, en ese lugar existió un puente que tuvo una longitud de 180 metros que se apoyaba en dos pilas centrales, fue un puente colgante que comunicó la ciudad y los campos agrícolas de la margen contraria del río en lo que ahora es Guatemala, argumentando que durante 700 años fue el puente más largo del mundo. Para comprobar esta hipótesis, faltaría encontrar los vestigios de la otra pila y analizar si realmente eran de concreto como propone el señor O'kon (es importante señalar que algunos investigadores no están de acuerdo con esta hipótesis). El caso de Copán es similar al de Yaxchilán, por lo tanto, este tipo de investigaciones también deberían realizarse en este sitio.

Este breve resumen acerca de las construcciones Mayas, tiene por objeto resaltar que la gran variedad y cantidad de construcciones realizadas fueron posibles utilizando tres materiales y un elemento fundamental: estucos, morteros y concretos, y el material fundamental el cemento Maya.

\section{Estucos, Morteros y Concretos}

El cemento en estado puro lo utilizaban los Mayas para fines decorativos en forma de estuco. Para usos estructurales fabricaban morteros y concretos, agregando a su cemento arenas y gravas calizas. Las gravas observadas en las muestras obtenidas están bien graduadas. La dimensión máxima de dichas gravas es aproximadamente de $5.5 \mathrm{~cm}$, con un promedio de $4 \mathrm{~cm}$. Los concretos obtenidos, son de 
color ocre blanquecino, es un material compacto y no presenta demasiadas oquedades por el proceso de colado (vaciado). El concreto fue utilizado en forma variada y en cantidades masivas. Se calcula que $1 / 16$ del total de las estructuras Mayas son de mortero y concreto, tan solo en la ciudad de Tikal en Guatemala, existen aproximadamente 3 000 edificios repartidos en una area de $16 \mathrm{Km}^{2}$ y cuyas edificaciones pueden alcanzar alturas de $72 \mathrm{~m}$. Pero Tikal no es la única ciudad de esta magnitud, existen otras de igual tamaño y decenas con dimensiones más modestas, pero de igual o mayor calidad arquitectónica; por ejemplo, Uxmal, Palenque, Calakmul por mencionar algunas. Al tomar en cuenta estos datos se puede tener una idea de la importancia que tuvieron en aquella época estos materiales.

\section{El cemento Maya}

Existen estructuras que para su construcción acabado o decoración utilizaron estuco, basado en cal como material cementante, mucho antes de que los Mayas lo utilizaran en sus grandes ciudades, por ello es difícil hablar de un "Cemento Maya". Cabe mencionar aquí la estructura "E-VII-sub" del conjunto del Uaxactún en el Petén, construida aproximadamente un siglo antes de Cristo y que conserva todavía algunos rasgos de la cultura Olmeca, sobre todo el detalle de los mascarones que representan cabezas de jaguares (Morley, 1972 y Soustelle, 1990). Esta estructura está totalmente revestida de un duro estuco, esto sucedió antes de que los Mayas empezaran su período de mayor grandeza, el clásico. Surge así el cuestionamiento del uso de cementantes como una aportación Olmeca a la naciente cultura Maya, para ligar los muros de mampostería o construir mascarones, sería entonces más apropiado referirse al cemento utilizado por los Mayas al considerar dicho material. Es un hecho comprobado que este cemento fue utilizado por ellos de muy diversas formas, desde las más finas en estucos para crear bellos y delicados tableros de fachadas o interiores, hasta efectivos y durables concretos que utilizaban en muros y columnas. Por lo tanto, si los Mayas no inventaron su propio cemento le dieron más versatilidad y lo enriquecieron con algunos agregados para usos verdaderamente estructurales. Esto es lo que se sabe hasta ahora, a no ser que los próximos descubrimientos arqueológicos revelen otra cosa.

La abundancia de rocas calizas en todos los asentamientos Mayas facilitó de cierta manera su fabricación, pues en la composición de todo cemento, incluido el moderno cemento Portland pasando por el cemento romano, aparece como componente principal la calcita $\left(\mathrm{CaCO}_{3}\right)$.

No se conocen referencias acerca de cómo fabricaban dicho cemento, solamente cómo fabricaban cal; incluso en
Yucatán todavía se obtiene de la misma manera que la obtenían los antiguos Mayas. Morley (1972), describe este procedimiento en tres pasos: a) la colocación de leños en forma radial para formar un círculo de 3 a $6 \mathrm{~m}$ de diámetro y $1.25 \mathrm{~m}$ de espesor, dejando un orificio central de $0.30 \mathrm{~m}$ de diámetro, b) la colocación de fragmentos de piedras calizas con espesor aproximado de $0.60 \mathrm{~m}$ sin llegar al perímetro exterior del círculo de leña y c) se prendía fuego desde el orificio central con hojas secas y madera podrida, con ello se lograba un proceso lento ( 36 hrs.) y continuo de quemado de abajo hacia arriba y de adentro hacia fuera. El mismo autor menciona que tenían la precaución de hacerlo cuando no había viento para que el quemado fuera homogéneo y tenían la creencia de que la presencia de mujeres era perjudicial para el proceso, por lo que evitaban que permanecieran cerca.

\section{Composición química del cemento}

Una cuestión por resolver, fue conocer si la matriz cementante de los estucos, morteros y concretos es la misma. El primer análisis que se llevó a cabo, consistió en determinar la composición química de la matriz cementante en estucos, morteros y concretos provenientes de tres diferentes lugares: Palenque, Yaxchilán y 'î̀niná. La figura 3, 4 y 5 ilustran ejemplos típicos de este tipo de materiales. Los microanálisis se obtuvieron utilizando un espectrómetro de rayos X (EDS) acoplado a un microscopio electrónico de barrido. Los resultados obtenidos se ilustran en la tabla 1. Esta tabla sugiere que no existen diferencias químicas apreciables entre las matrices cementantes de los estucos, morteros y concretos mayas. En esta tabla se presentan también, los análisis químicos en gravas calizas, representativas de las rocas utilizadas para la obtención de la cal. Se observa en las gravas, aproximadamente el mismo porcentaje que los contenidos en la matriz cementante. En la última columna de esta tabla se ilustra la composición de un cemento Portland comercial; sin embargo, la comparación con los porcentajes ilustrados en las otras columnas no sugiere ningún tipo de conclusiones sobre la naturaleza de las diferentes matrices cementantes y el cemento Portland.

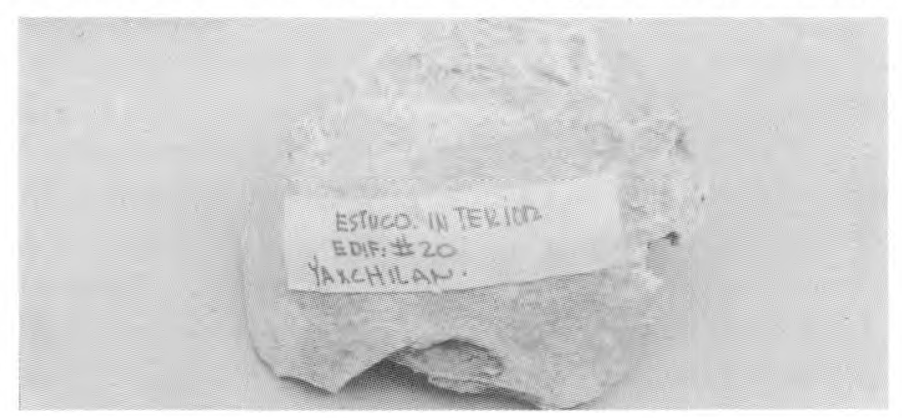

Figura 3. Muestra de estuco proveniente de Yaxchilán 


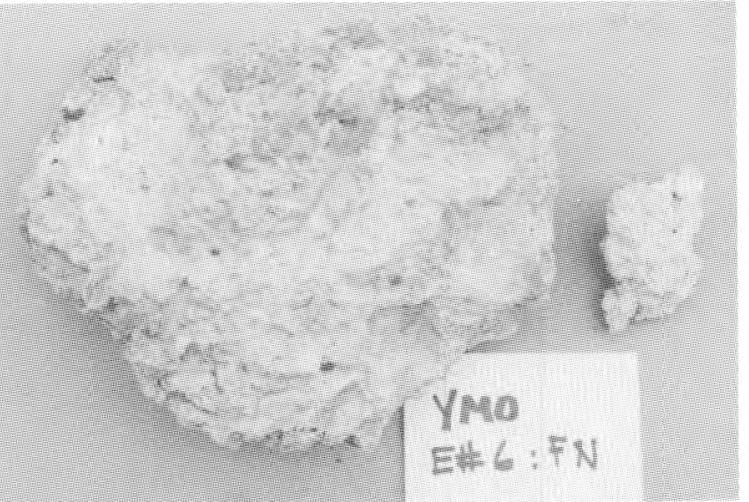

Figura 4. Muestra de mortero proveniente de Yaxchilán

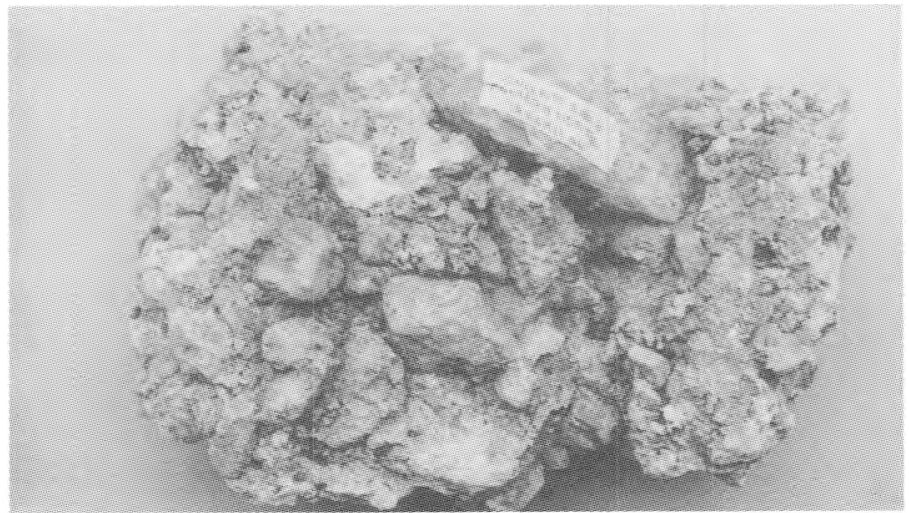

Figura 5. Muestra de concreto proveniente de Yaxchilán

Tabla 1. Composición química promedio de la matriz cementante en estucos, morteros, concretos, gravas calizas y cemento Portland. No se aprecian diferencias en los valores obtenidos

\begin{tabular}{cccccc}
\hline \hline & Estuco & Mortero & Concreto & Grava & $\begin{array}{c}\text { Cemento } \\
\text { Portland }\end{array}$ \\
\hline $\mathrm{C}$ & 23.59 & 20.02 & 13.17 & 19.22 & 10.66 \\
$\mathrm{O}$ & 39.64 & 23.73 & 38.42 & 42.72 & 46.24 \\
$\mathrm{Na}$ & - & - & - & - & 3.31 \\
$\mathrm{Mg}$ & 5.75 & 1.57 & 5.48 & 3.48 & 1.37 \\
$\mathrm{Al}$ & 4.11 & 3.84 & 1.17 & 1.67 & 2.70 \\
$\mathrm{Si}$ & 4.20 & 6.65 & 2.82 & 3.07 & 9.59 \\
$\mathrm{~S}$ & - & - & - & - & 1.30 \\
$\mathrm{~K}$ & - & 0.99 & - & - & 0.92 \\
$\mathrm{Ca}$ & 19.06 & 36.97 & 38.01 & 28.44 & 22.66 \\
$\mathrm{Fe}$ & 3.65 & 4.87 & - & 0.71 & 1.25 \\
$\mathrm{P}$ & - & 1.35 & - & 0.69 & - \\
$\mathrm{Y}$ & - & - & 0.94 & - & - \\
TOTAL & 100.00 & 100.00 & 100.00 & 100.00 & 100.00 \\
\hline
\end{tabular}

Nota: Los altos contenidos de carbono están principalmente relacionados con problemas de contaminación de la ventana del detector.
Por otro lado, no se encontraron diferencias apreciables entre las composiciones químicas de estucos, morteros y concretos que provenían de diferentes lugares. Por ejemplo, la figura 6 , ilustra esquemáticamente tres diferentes espectros de composición (EDS), obtenidos a partir de muestras de estucos de diferentes lugares tales como: Palenque (A), Yaxchilán (B) y Toniná (C). Esta figura ilustra claramente que la composición de la matriz cementante es similar en los tres tipos de muestras.

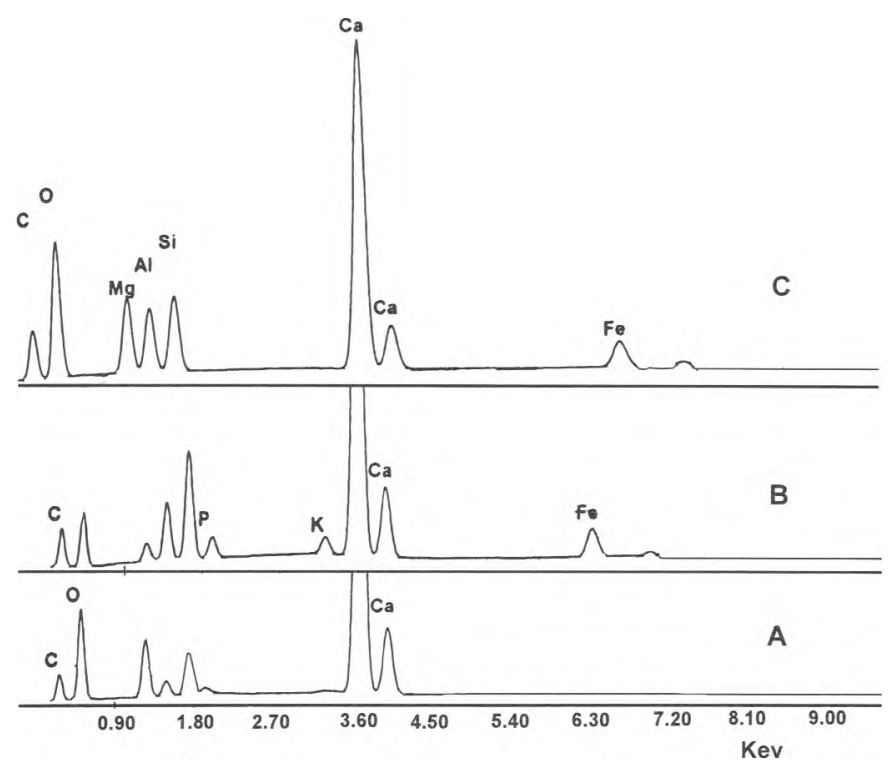

Figura 6. Espectros de composición química (EDS) obtenidos a partir de muestras de estucos provenientes de diferentes regiones. $A$ (Palenque), B (Yaxchilán) y C (Toniná). La composición química de la matriz cementante de estas 3 regiones es muy similar.

Sin embargo, cuando se determinaron los óxidos presentes en las matrices cementantes y en los cementos comerciales, se encontraron diferencias apreciables. Este tipo de análisis se obtuvo utilizando un espectrómetro de arco y chispa. Los resultados obtenidos se ilustran en la tabla 2. Existen diferencias apreciables entre los contenidos de $\mathrm{SiO}_{2}, \mathrm{Al}_{2} \mathrm{O}_{3}$ y $\mathrm{Fe}_{2} \mathrm{O}_{3}$ en los cementos comerciales en comparación con las matrices cementantes, principalmente en el caso del $\mathrm{SiO}_{2}$, en donde los contenidos en la matriz cementante son muy bajos en comparación con los contenidos en el cemento Portland. Similares resultados se obtienen para $\mathrm{Al}_{2} \mathrm{O}_{3}$ y $\mathrm{Fe}_{2} \mathrm{O}_{3}$, aunque en estos casos las diferencias no son tan pronunciadas. 
Tabla 2. Contenido de óxidos en la matriz cementante de morteros provenientes de Yaxchilán, Palenque, Comal. caco, Toniná y también en los cementos Portland y

Portland hidratado

\begin{tabular}{|c|c|c|c|c|}
\hline & $\mathrm{Al}_{2} \mathrm{O}_{3} \%$ & $\mathrm{SiO}_{2} \%$ & $\mathrm{CaO} \%$ & $\mathrm{Fe}_{2} \mathrm{O}_{3} \%$ \\
\hline Cemento Portland & $5.90 \pm .0 .08$ & $29.9 \pm 2.9$ & $51.7 \pm 3.0$ & $2.4 \pm 0.3$ \\
\hline $\begin{array}{c}\text { Cemento Portland } \\
\text { (hidratado) }\end{array}$ & $4.80 \pm 0.07$ & $23.84 \pm 2.4$ & $41.8 \pm 2.0$ & $2.0 \pm 0.2$ \\
\hline Mortero (Yaxchilan) & $1.8 \pm 0.02$ & $2.5 \pm 0.02$ & $43.1 \pm 2.0$ & $0.54 \pm 0.06$ \\
\hline Mortero (Palenque) & $1.4 \pm 0.02$ & $3.7 \pm 0.3$ & $40.8 \pm 2.0$ & $0.49 \pm 0.05$ \\
\hline Mortero (Comalcalco) & $2.5 \pm 0.02$ & $4.3 \pm 0.4$ & $47.6 \pm 3.0$ & $0.73 \pm 0.08$ \\
\hline Mortero (Toniná) & $0.8 \pm 0.008$ & $3.4 \pm 0.3$ & $36.5 \pm 2.0$ & $1.0 \pm 0.1$ \\
\hline
\end{tabular}

Para determinar las fases cristalinas presentes en las matrices cementantes de estucos, morteros, concretos y también en el cemento Portland, se obtuvieron patrones de difracción de rayos X. La figura 7 ilustra el espectro de composición química (EDS) con su respectivo patrón de difracción, obtenidos a partir de la matriz cementante de una muestra de estuco (Palenque). En la figura 8 se ilustran espectros similares (de composición química y de difracción de rayos X) para una muestra de cemento Portland comercial. La comparación entre los difractogramas sugiere diferencias apreciables entre las fases cristalinas presentes en ambos compuestos. Esto se ilustra claramente en la figura 9 donde se presentan 3 patrones de difracción. El primer espectro (A) fue obtenido a partir de muestras de cemento Portland. Los otros dos espectros ( $\mathrm{B}$ y $\mathrm{C}$ ) fueron obtenidos a partir de las matrices cementantes de muestras de estucos provenientes de Palenque y Yaxchilán. El patrón de difracción mostrado en la figura 9, indica la presencia de silicatos cálcicos $\left(\mathrm{Ca}_{3} \mathrm{SiO}_{5}\right)$ cuyos principales picos son indicados por el número "2". Los espectros mostrados en las figuras $9 \mathrm{~B}$ y $9 \mathrm{C}$ indican principalmente la presencia de calcita $(\mathrm{CaCO} 3)$ cuyos correspondientes picos son indicados con el número "1". Estos resultados muestran que la principal diferencia entre el cemento Portland y la matriz cementante de los estucos Mayas está relacionada con la presencia de compuestos de silicio (silicato) en el cemento Portland y la completa ausencia de estos compuestos en las matrices cementantes Mayas.

\section{Propiedades mecánicas del concreto}

Indicadores de las propiedades mecánicas en concretos de origen Maya se han obtenido a partir de ensayos de compresión simple y de velocidad de pulso. Por las restricciones reglamentarias del INAH, las muestras de concreto que se han podido obtener no corresponden a materiales sanos, por lo tanto, son pocas las probetas que se han podido
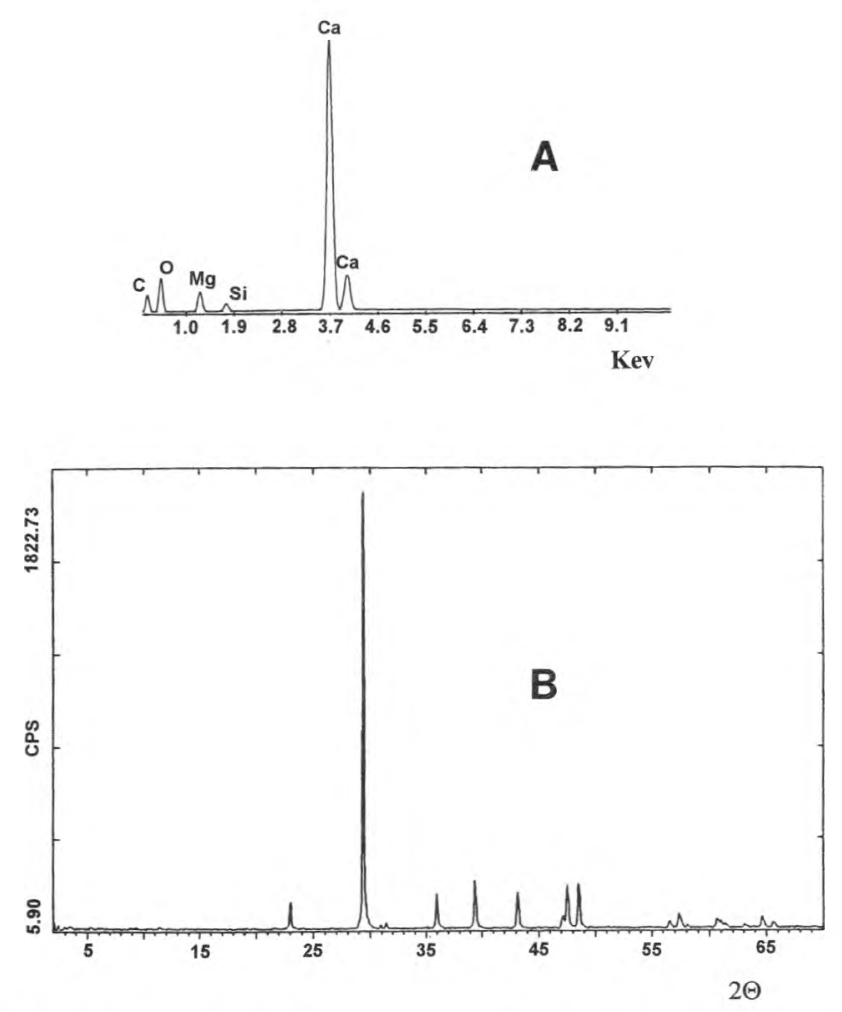

Figura 7. A) Espectro de composición química (EDS) obtenido de la matriz cementante de un estuco proveniente de Palenque. B) Patrón de difracción obtenido de esta muestra de estuco. Los picos mostrados en este patrón corresponden a la calcita $\left(\mathrm{CaCO}_{3}\right)$
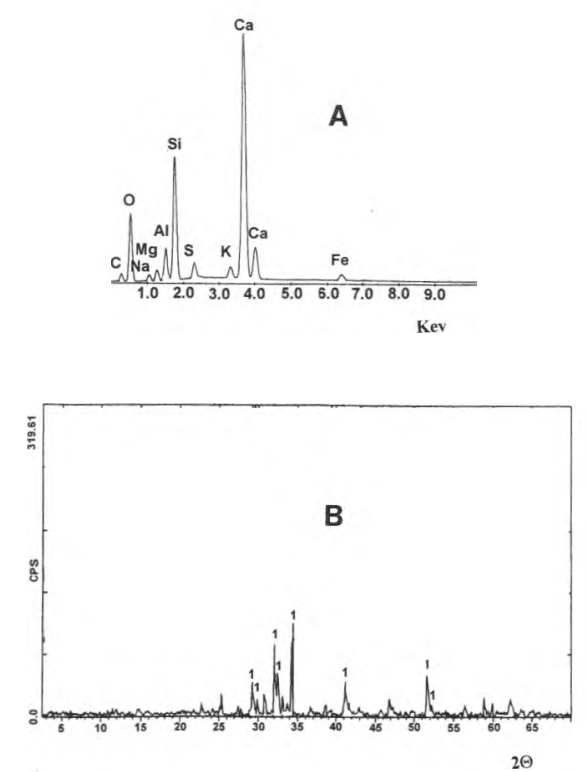

Figura 8. A) Espectro de composición química (EDS) obtenido de una muestra de cemento Portland comercial. B) Patrón de difracción correspondiente a este tipo de cemento. Los picos del patrón indicados con el número " $I$ " correponden a un silicato cálcico $\left(\mathrm{Ca}_{3} \mathrm{SiO}_{5}\right)$. 


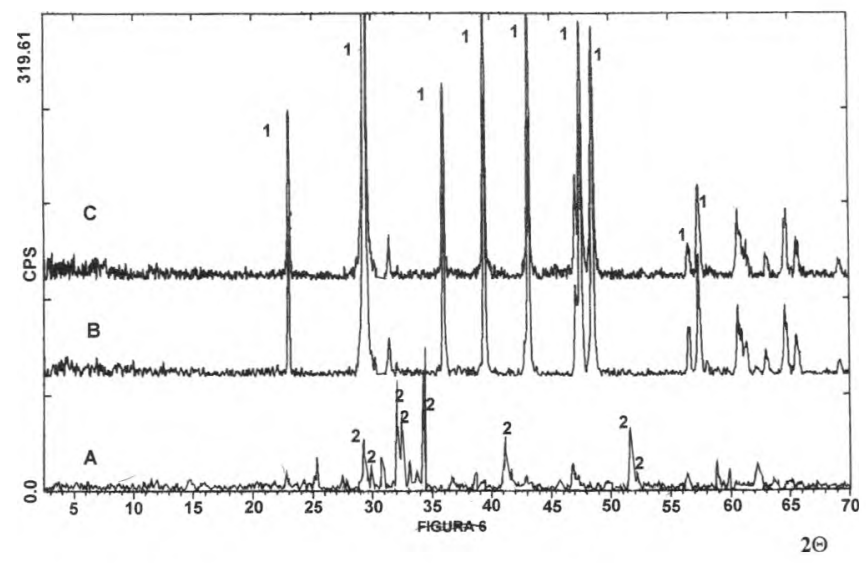

Figura 9. A) Patrón de difracción de una muestra de cemento Portland. Los picos asociados al número " 2 " indican la presencia de silicatos cálcicos $\left(\mathrm{Ca}_{3} \mathrm{SiO}_{5}\right)$. B) y C) Patrones de difracción obtenidos de la matriz cementante en estucos provenientes de Palenque y Yaxchilán. Los picos de estos patrones indicados con el número "I" corresponden a la calcita $\left(\mathrm{CaCO}_{3}\right)$

obtener. Los resultados que se presentan en la tabla 3, corresponden a probetas labradas de muestras de concreto de Yaxchilán, provenientes de un coronamiento colapsado del Edificio No 6. Aún cuando las probetas preparadas para estas pruebas no se apegan completamente a las especificaciones aplicables, los valores obtenidos dan una idea del comportamiento esperado. La velocidad de pulso obtenida es mas baja que en los concretos actuales, además hay un cambio importante de este parámetro entre las direcciones ortogonales ensayadas, lo cual se puede relacionar con el grado de daños y agrietamiento interno de las probetas. El promedio del peso volumétrico obtenido es de $1.83 \mathrm{gr} / \mathrm{cm}^{3}\left(17.9 \mathrm{kN} / \mathrm{m}^{3}\right)$, que resulta comparable al del concreto, moderno con agregados andesíticos o similares. El promedio de resistencia obtenido es de $66 \mathrm{~kg} / \mathrm{cm}^{2}(6.5 \mathrm{Mpa})$ que resulta inferior al común de los concretos modernos; sin embargo, resulta suficiente para los fines estructurales originales, en los cuales se han estimado esfuerzos actuantes de no más de $25 \mathrm{~kg} / \mathrm{cm}^{2}$ ( $2.4 \mathrm{Mpa}$ ). Es de esperarse resistencias mayores en muestras de materiales más sanos. De hecho, esto ya se ha comprobado, ya que ensayos hechos recientemente en muestras de mejor consistencia arrojan resistencias de 140 a 170 $\mathrm{kg} / \mathrm{cm}^{2}$ (13.7-16.7 Mpa). También se han obtenido valores de resistencia a la tensión, por medio del módulo de ruptura a flexión, resultando de $18 \mathrm{~kg} / \mathrm{cm}^{2}$ (1.8 Mpa) en promedio.

Tabla 3. Medidas de resistencia a la compresión simple en muestras de concretos provenientes de 3 zonas diferentes. I (Palenque), II (Yaxchilán) y III (Toniná)

\begin{tabular}{|c|c|c|c|c|}
\hline PROBETA & \multicolumn{2}{|c|}{$\begin{array}{l}\text { VELOCIDAD DE PULSO } \\
\text { DIR.X } \quad \text { DIR.Y }\end{array}$} & $\begin{array}{c}\text { PESO } \\
\text { VOLUMETRICO } \\
\mathrm{gr} / \mathrm{cm}^{3}\left(\mathrm{kN} / \mathrm{m}^{3}\right)\end{array}$ & $\begin{array}{l}\text { RESISTENCIA } \\
\text { COMPR.SIMPL. } \\
\mathrm{kg} / \mathrm{cm}^{2}(\mathrm{MPa})\end{array}$ \\
\hline I & $53.0 \times 10^{5}$ & $34.9 \times 10^{5}$ & $1.87(18.3)$ & $58(5.7)$ \\
\hline II & $52.8 \times 10^{5}$ & $32.6 \times 10^{5}$ & $1.82(17.8)$ & $73(7.2)$ \\
\hline III & $51.2 \times 10^{5}$ & $31.0 \times 10^{5}$ & $1.80(17.6)$ & $60(5.9)$ \\
\hline
\end{tabular}

\section{Aplicaciones estructurales}

Es difícil encontrar alguna estructura Maya en donde no estén presentes el estuco, el mortero y el concreto. Los estucos se utilizaban para construir tableros de relieves en fachadas exteriores e interiores, o como acabado fino en muros, comúnmente para construir sus mascarones. Los morteros y concretos tenían en las construcciones Mayas un uso estructural. Los morteros se utilizaban como material de liga en las mamposterías para formar muros en los edificios y para revestir sus cisternas y caminos, también se utilizaban como material de liga en sus arcos (Korbeles) y bóvedas, construidas a base de losas saledizas (ver figura 1, que muestra un ejemplo notable del arco Maya). Para la construcción de las losas (viga-losa), Morley (1972) menciona que el método que se utilizaba consistía primero en colocar un sistema de vigas de madera como elementos de apoyo, enseguida se construía un entramado de madera que funcionaba como cimbra, en donde se vaciaba el concreto, esperaban el tiempo necesario, y retiraban entonces el entramado de madera quedando la losa de concreto.

\section{Conclusiones}

El estuco, los morteros y concretos fueron ampliamente utilizados por la cultura Maya. Todos estos materiales utilizaban el mismo tipo de matriz cementante. Los análisis químicos de la matriz cementante muestran la carencia de silicatos y aluminatos en el cemento Maya, por lo que estamos en presencia de un material aglutinante con fuertes propiedades cementantes y con una composición semejante a un cemento natural "crudo". La quemada y molida de rocas calizas con porcentaje variable de intrusiones de material arcilloso fue 
probablemente la técnica de su fabricación. Los hornos utilizados en este proceso, no superaban a los $900{ }^{\circ} \mathrm{C}$, que es la temperatura necesaria para que las rocas calizas pierdan su dióxido de carbono.

Esto explica también la carencia de silicatos, pues estos requieren temperaturas mínimas de aproximadamente 1100 C. Por esta razón, este tipo de compuestos es facilmente encontrado en el cemento Portland. El cemento Maya para fines decorativos se aplicaba en estado puro y para usos estructurales se enriquecía con agregados, arenas y gravas calizas para fabricar morteros y concretos.

\section{Agradecimientos}

Los autores expresan su agradecimiento al CONACYT por su apoyo económico, así como al Instituto Nacional de Investigaciones Nucleares (ININ) y al Instituto de Antropología e Historia por el uso de su infraestructura técnica.

\section{Referencias}

Morley G.S. (1972). La civilización Maya. F.C.E. 2 da. Edición, México.
O'kon J.A. (1995). Bridge to the Past, Civil Engineering, Enero.

Rivera-Dorado M. (1985). Los Mayas de la antigüedad, Madrid, Alhambra.

Soustelle J. (1990). Los Mayas, Edit. F.C.E. México.

\section{Bibliografía sugerida}

Díaz-Bolio J. (1992). The Mayan Magnifecent Ancient White Roads, Area Maya, Mérida Yucatán.

Flores-Jiménez R. (1978). Cultura Maya, Biblioteca Nacional de Antropología e Historia, INAH.

Lea (1993). The Chemistry of Cement and Concret, Mc. Graw Hill, México.

Neville A.M. (1977). Tecnología del concreto I,II , Instituto Mexicano del Cemento y del Concreto AC.

Romero R.M.E. (1998). Revista arqueológica mexicana, No. 33, volumen VI.

Thompsom J.E. (1985). Grandeza y decadencia de los Mayas, F.C.E., México.

Von-Hagen V.M. (1960). El Mundo de los Mayas, Editorial Diana, 5 ta. Edición, México.

\section{Semblanza de los autores}

Ramiro Pérez-Campos. Realizó su licenciatura en física y matemáticas en la Escuela Superior de Física y Matemáticas del IPN, realizó su doctorado en la Universidad de Alberta, Canadá. Es investigador titular "C" del Centro de Ciencias Físicas y actual coordinador del Programa de Investigación y Desarrollo de Ductos del Instituto Mexicano del Petóleo. Es investigador Nacional nivel 3 (SNI). Sus ramas de investigación son la microscopía electrónica, metalurgia y ciencia de materiales.

Heriberto Díaz-Coutiño. Es profesor de tiempo completo en la Facultad de Ingeniería Civil de la Universidad Autónoma de Sinaloa. Se doctoró en la Facultad de Ingeniería de la Universidad Autónoma del Estado de México con la tesis: La matriz cementante en la cultura maya.

Horacio Ramírez de Alba. Realizó sus estudios de ingeniería civil en la Facultad de Ingeniería de la Universidad Nacional del Estado de México; obtuvo el grado de maestro en ingeniería en la UNAM y de doctorado en la Universidad de Texas en Austin. Es especialista en ingeniería estructural y ha realizado algunos trabajos en materiales prehispánicos. 